\title{
Opportunities in a social world
}

\author{
Social networks - what are they good for? We don't know for sure, but we're keen to find out.
}

Early in 2011, we decided it was time for Nature Physics to take its first tentative steps into the uncertain world of social media, and began using Twitter (under the handle@NaturePhysics). We didn't know exactly what we, or the many thousand followers we'd accumulated since reserving the name, wanted from it. But we've got some ideas, and we're hoping that you will join us on the journey.

A recent study of the social graph of Facebook (L. Backstrom et al., http:// arxiv.org/abs/1111.4570; 2011) found that any two of its more than 700 million users are separated on average by just 3.74 intermediate users. The efficiency with which it is possible to connect and interact with your social network is nothing short of revolutionary. In the realm of politics, this is often literally so. And in physics it has the potential to be a 'disruptive technology', to say the least.

Preprints of many scientific papers (and in some disciplines, most) are now posted as a matter of course to the arXiv preprint sever at the same time as they are submitted for formal publication. The advent of social networks means that the most potentially exciting and certainly controversial of these papers can be launched into public view well before they are formally published, let alone peer reviewed. This presents challenges and opportunities.

Peer review is a cornerstone of the scientific method. And as imperfect as it might be, we feel it is still the least flawed of the alternatives so far. Yet, social networks and technologies have the potential to enable new results to be scrutinized more quickly, by more people and with more openness than has ever been possible. For instance, remember the September 2011 announcement by the OPERA collaboration of results suggesting that neutrinos might travel at superluminal speeds (http://arxiv.org/abs/1109.4897; 2011): within just two weeks, dozens of technical papers, blogposts and tweets pawing over the results, suggesting sources of uncertainty and error, and contemplating their implications, had been written.

Many might prefer that these discussions remain within the cloistered corridors of academia, so that a consensus can be reached before the results are released to an unprepared public. But only a matter of hours had passed after the OPERA neutrino-anomaly preprint had been posted to the arXiv preprint server before The New York Times posed the question, "Roll over, Einstein?" (http://www.nytimes.com/2011/09/23/ science/23speed.html). And a day later the same publication reported the flood of scrutiny in the blogosphere that followed. This is science in the open. And it's just the beginning.

How is the scientific community to respond? It's too late to put the genie back in the bottle, the only option is to engage. And there are now many places to do so. To share news and comment that we come across in our travels around the Internet, we'll continue to use Twitter. But for more detailed comment and discussion, we now have a page on Google+. Where it will lead, it is too soon to tell. But we hope you'll join us in finding out together.

Join in the conversation on Twitter at http://twitter.com/NaturePhysics.

Join in the conversation on Google+ at http://gplus.to/NaturePhysics.

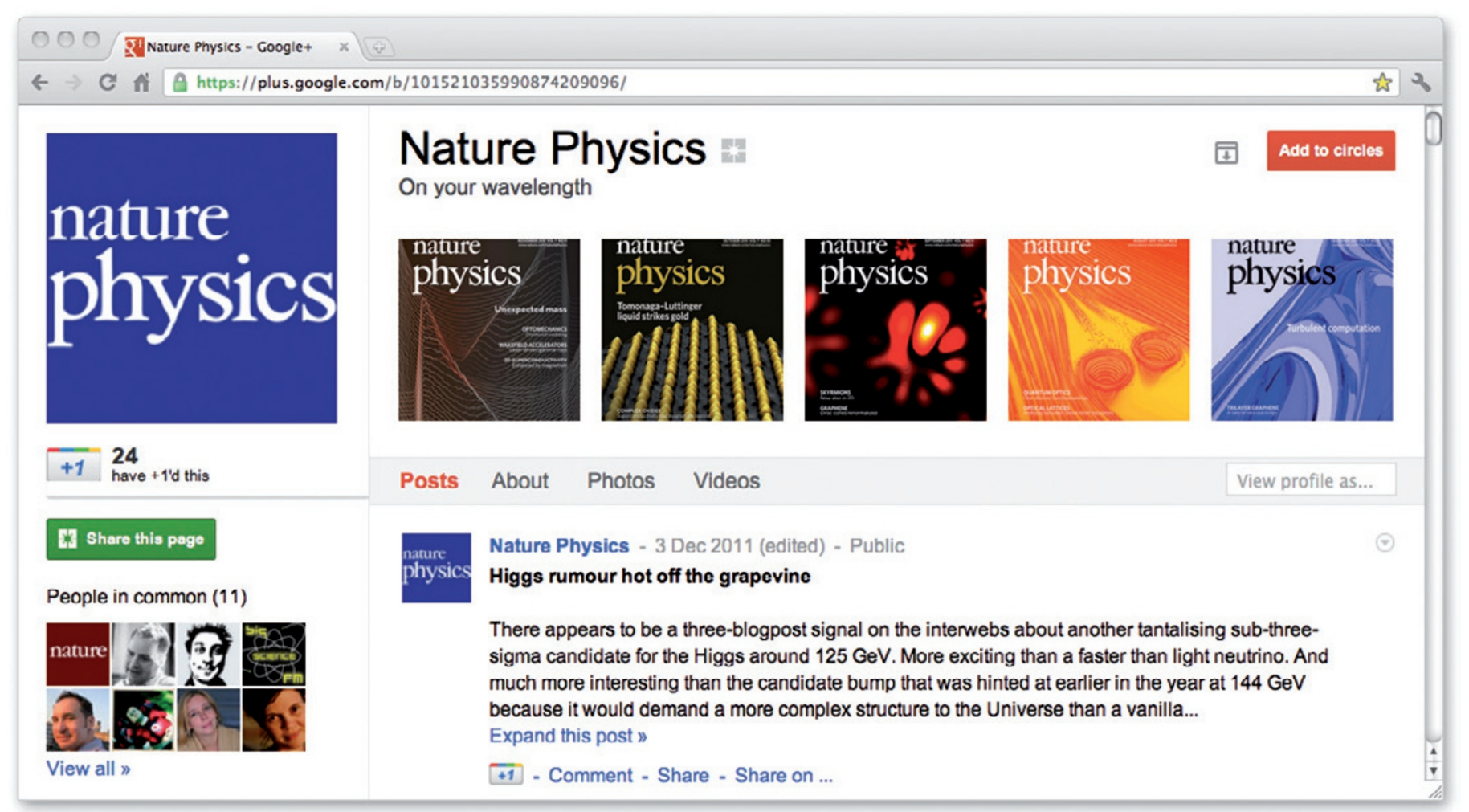

